Revue de droit comparé du travail et de la sécurité sociale

2| 2018

Harcèlement moral au travail en jurisprudence

comparée

\title{
La jurisprudence australienne sur le harcèlement psychologique
}

Peter Hampton

\section{OpenEdition}

Journals

Édition électronique

URL : https://journals.openedition.org/rdctss/1948

DOI : $10.4000 /$ rdctss. 1948

ISSN : 2262-9815

Éditeur

Centre de droit comparé du travail et de la sécurité sociale

Édition imprimée

Date de publication : 1 juin 2018

Pagination : 58-69

ISSN : $2117-4350$

Référence électronique

Peter Hampton, «La jurisprudence australienne sur le harcèlement psychologique », Revue de droit comparé du travail et de la sécurité sociale [En ligne], 2 | 2018, mis en ligne le 01 novembre 2021, consulté le 13 novembre 2021. URL : http://journals.openedition.org/rdctss/1948 ; DOI : https:// doi.org/10.4000/rdctss. 1948

\section{(c)}

Revue de droit comparé du travail et de la sécurité sociale est mise à disposition selon les termes de la Licence Creative Commons Attribution - Pas d'Utilisation Commerciale - Pas de Modification 4.0 International. 


\section{LA JURISPRUDENCE AUSTRALIENNE SUR LE HARCÈLEMENT PSYCHOLOGIQUE}

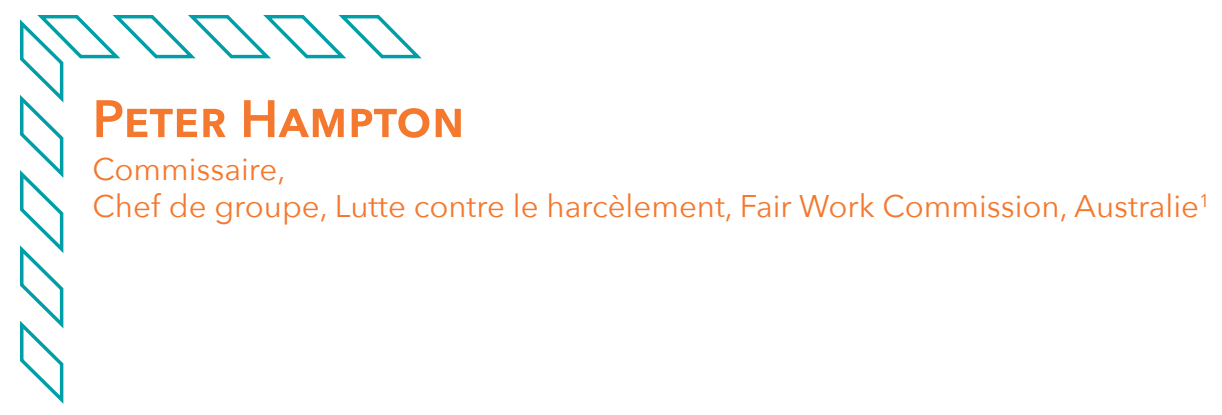

'Australie a une longue tradition de protection contre le harcèlement psychologique, également appelé « harcèlement au travail ». Pendant de nombreuses années, les lois au niveau des Etats et, plus tard, au niveau fédéral, ont protégé les travailleurs contre les risques pour leur santé et leur sécurité découlant des risques professionnels ${ }^{2}$. Cela s'étend au harcèlement sur le lieu de travail, qui, dans ce contexte, est le comportement d'un ou de plusieurs individus contre un ou plusieurs travailleurs qui crée un risque réel ou apparent pour la santé ou la sécurité psychologiques de cette personne. Cet article examinera brièvement l'application et les limites potentielles du droiten matière de santé et de sécurité au travail.L'Australie dispose également de systèmes relativement complets prévoyant une indemnisation des travailleurs, notamment en raison de dommages psychologiques. Ce droit, ainsi que les droits connexes de Common law en matière de dommages-intérêts pour le comportement négligent d'autrui, sont des éléments importants du système australien, cependant, la nécessité dicte que l'accent soit mis dans cette contribution sur le droit jurisprudentiel.

Un ajout important aux options en matière de prévention est apparu relativement récemment en raison d'une jurisprudence novatrice qui permet aux individus de faire sanctionner les problèmes de harcèlement en milieu de travail dans le but de contrer l'intimidation au travail qui constituerait un risque

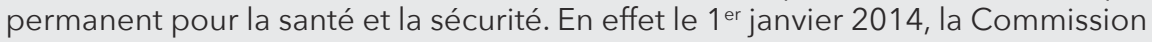
du travail équitable (" la Commission ci-après»), c'est-à-dire le tribunal national des relations de travail, a été dotée de pouvoirs spécifiques pour traiter les

1 Les opinions de l'auteur soutenues dans cette contribution ne sont pas celles de la Fair Work Commission.

2 Commençant par la législation sur les usines, traitant principalement des dangers physiques et des conditions de travail et s'étendant plus directement aux considérations de santé et de bien-être depuis le début des années 1970. 


\section{AUSTRALIE}

demandes en matière de harcèlement au travail. Cet article se penchera sur le contexte dans lequel cette nouvelle compétence a été instituée, sur les autres juridictions qui restent également accessibles aux parties, et sur l'application au cours des quatre dernières années des nouvelles dispositions.

Avant l'arrivée des Européens, les Australiens autochtones avaient un système sophistiqué de lois et de codes non écrits ${ }^{3}$. Le système juridique australien moderne a été largement inspiré de celui de la Grande-Bretagne, avec un système de gouvernement de type «Westminster ", la création de tribunaux séparés et indépendants pour traiter de l'application, de l'interprétation et de l'exécution des lois adoptées par le Parlement, et le concept de la Common law (droit des juges ou loi jurisprudentielle ${ }^{4}$ ) établissant des principes juridiques et des droits qui fonctionnent sous réserve de l'application de la loi. Ce modèle a été adopté dans chacune des colonies d'Australie.

L'Etat fédéral du Commonwealth d'Australie a été formé en 1900 avec l'adoption par le gouvernement du Royaume-Uni du Commonwealth of Australia Constitution Act 1900 ( "la Constitution »). Lors de la création de la Fédération, les colonies sont devenues des Etats, chacun d'entre eux conservant son propre Parlement, sa Constitution et la compétence de légiférer en général. La Constitution établit le cadre du système juridique australien et une liste de questions, ou "chefs de compétence ", sur lesquels le gouvernement du Commonwealth peut légiférer ${ }^{5}$. Lorsqu'une loi d'un Etat est incompatible avec une loi du Commonwealth, c'est la loi du Commonwealth qui s'impose en général ${ }^{6}$.

Depuis la création de l'Etat fédéral, ces chefs de compétence ont fait l'objet d'interprétations, avec un élargissement progressif de leur champ d'application. Les sujets abordés dans cet article illustrent bien cette tendance; traditionnellement les questions relatives à la santé et à la sécurité au travail, y compris le harcèlement psychologique, ont été du ressort des Etats. Plus récemment, cependant, la Constitution a été utilisée pour élargir la portée des lois du Commonwealth à un nombre croissant de questions liées au lieu de travail, dont le harcèlement, en s'appuyant sur la compétence fédérale pour règlementer les sociétés ${ }^{7}$.

\section{I - L'INDEMNISATION DES CONSÉOUENCES DU HARCÈLEMENT AU TRAVAIL}

\section{A - Les ACtIONS EN COMMON LAW}

Les principes de Common law en matière de rupture de contrat peuvent s'appliquer pour réparer le préjudice subi par une victime de harcèlement au travail. Le harcèlement, en tant que violation du contrat de travail, peut être retenu lorsque l'employeur n'a pas

3 C'est un sujet à part entière, notamment en ce qui concerne la poursuite des traditions et coutumes anciennes.

4 Voir la discussion dans Lipohar v The Queen (1999) 200 CLR 485.

5 Commonwealth of Australia Constitution Act 1901 s 51.

6 Commonwealth of Australia Constitution Act 1901 s 109.

7 Discuté dans State of New South Wales v Commonwealth of Australia (Work Choices Case) [2006] HCA 52. 
respecté une stipulation expresse ou implicite du contrat de travail. Une stipulation expresse peut s'étendre à certaines politiques et procédures incorporées dans un contrat de travail ${ }^{8}$.

Il y a aussi certaines stipulations dans le contrat de travail qui sont implicites soit de fait, soit en droit, c'est-à-dire qu'elles ne résultent pas d'une clause, mais relèvent " en fait » de l'intention des parties d'être liées par ces conditions, ou « en droit » lorsqu'elles se sont développées en vertu de la Common law. L'une de ces conditions implicites en droit est le devoir de l'employeur de prendre des mesures raisonnables pour assurer la sécurité des salariés ${ }^{9}$. Une illustration de cette obligation implicite est donnée dans une affaire récente, Keegan c. Sussan Corporation (Aust) Pty Ltd, dans laquelle une travailleuse a obtenu des dommages-intérêts et une indemnisation après avoir subi un préjudice psychologique parce qu'elle était victime de harcèlement au travail après son retour à la fin de son congé de maternité. Cette décision fait suite à un certain nombre de décisions importantes, concluant " qu'il est bien établi que le devoir de diligence de l'employeur comprend l'obligation de prendre toutes les mesures raisonnables pour fournir un système de travail sécurisé et de prendre des précautions raisonnables pour éviter les blessures psychologiques ${ }^{10} »$.

Les actions en relation avec la violation des termes exprès ou implicites du contrat de travail ne sont pas rares et peuvent entraîner le versement de dommages et intérêts considérables. Cependant, ce n'est pas une voie à laquelle la plupart des travailleurs peuvent accéder facilement en raison des restrictions légales, par exemple la compensation pour les préjudices subis au travail peut être limitée par le cadre légal d'indemnisation des travailleurs ${ }^{11}$, et aussi des coûts d'une procédure de cette nature.

\section{B - LES LOIS SUR L'INDEMNISATION DES TRAVAILLEURS}

Le Commonwealth et chaque Etatet territoire d'Australie ont un système d'indemnisation des travailleurs "sans faute $»^{12}$. En règle générale, cela permet à un travailleur blessé d'avoir accès à des indemnités pour perte de salaire et frais médicaux pour des blessures ou des maladies découlant de son emploi. Ce système est subordonné à des limites chronologiques et de montant. L'indemnisation s'étend aux réclamations pour préjudice psychologique, mais dans la plupart des Etats, de telles réclamations sont limitées aux cas où la blessure ne résulte pas d'une « action administrative (ou dans certains cas de gestion) raisonnable $»^{13}$.

8 Goldman Sachs JB Were Services Pty Ltd v Nikolich (2007)163 FCAFC 120.

9 Keegan $v$ Sussan Corporation (Aust.) Pty Ltd [2014] QSC 64 at [20], following Tame $v$ New South Wales (2002) 211 CLR 317, 365.

10 Keegan v Sussan Corporation (Aust.) Pty Ltd [2014] QSC 64 at [21], suivant Tame v New South Wales (2002) 211 CLR 317, 365, suivant Koehler v Cerebos (Aust) Ltd (2005) 222 CLR 44, 53.

11 Par exemple, l'article 44 de la Loi de 1988 sur la sécurité, la réadaptation et l'indemnisation prévoit que lorsqu'un salarié demande des dommages-intérêts pour perte non financière (douleur et souffrances), il ne peut prétendre à une indemnité pour accident et n'a droit qu'à un montant maximum de $\$ 110000$. D'autres lois sur l'indemnisation des travailleurs limitent ou éliminent de manière significative les actions en Common law, sauf dans le cas des blessures les plus graves.

12 Le premier régime en Australie était le Workers Compensation Act 1900 (Australie du Sud).

13 Par exemple, l'article 5A(1)(c) et(2) de la loi de 1988 sur la sécurité, la réadaptation et l'indemnisation exclut une maladie, une blessure ou une aggravation subie à la suite de mesures administratives raisonnables prises de manière raisonnable concernant l'emploi du salarié en question. 


\section{C - Les Autres ChEFS de COMPÉTENCES}

\section{Harcèlement et discrimination}

Il n'est pas possible dans le cadre de cette contribution de présenter dans le détail le harcèlement en milieu de travail lorsqu'il repose sur des motifs discriminatoires tels que le sexe, la race, le handicap, etc. Cependant, il est important de noter que lorsque le harcèlement dans le travail repose sur des attributs protégés par la législation anti discrimination, cela permet d'agir sur ce fondement que ce soit au niveau fédéral ou à celui des Etats et Territoires ${ }^{14}$. Ces dispositions impliquent l'application potentielle de sanctions contre les responsables et l'indemnisation des dommages.

De même, il existe également des dispositions spécifiques aux « actions préjudiciables » prises sur le lieu de travail qui couvrent un éventail de scénarios, en vertu de la Fair Work Act de 2009 du Commonwealth ${ }^{15}$. Ces actions constituent une violation des " protections générales » d'un salarié et comprennent la protection d'un droit défini ou de l'exercice de ce droit sur le lieu de travail, par exemple en se renseignant sur ses droits ou en adressant une demande à la Commission. Elles comprennent également les motifs discriminatoires généralement compris tels qu'énoncés ci-dessus. Les " actions préjudiciables » peuvent inclure la cessation d'emploi ou les "blessures dans le travail ", les deux pouvant être considérés comme du harcèlement au travail. Des pénalités et une indemnisation du requérant peuvent également être attribuées par la Cour.

\section{Règlementation de la santé et de la sécurité au travail}

Jusqu'à tout récemment, la réglementation de l'intimidation sur le lieu de travail relevait généralement de la seule responsabilité des organismes de réglementation de la santé et de la sécurité au travail au niveau de l'Etat et du gouvernement fédéral. Les gouvernements à chacun de ces niveaux ont légiféré pour la réglementation de la santé et de la sécurité au travail, les « régulateurs » étant les organes chargés d'appliquer cette législation, ce qu'ils font dans le cadre de leurs propres politiques et directives.

La réglementation de la santé et de la sécurité sur le lieu de travail en Australie a débuté avec l'adoption de la loi Factories and Shops Act de 1885 (loi sur les fabriques et les magasins) dans l'Etat de Victoria, une législation similaire étant adoptée par la suite dans d'autres Etats. Cette législation visait en grande partie à réglementer des conditions de travail spécifiques, notamment pour les femmes et les enfants dans les usines et les commerces de détail. La Constitution australienne ne prévoit pas directement que le gouvernement du Commonwealth légifère en matière de santé et de sécurité au travail et, jusqu'à récemment, cette compétence relevait des Etats et Territoires. En 1991, le Commonwealth a légiféré en ce qui concerne la santé et la sécurité de ses propres salariés et cela a ensuite été étendu à d'autres éléments du secteur privé en Australie en utilisant d'autres chefs de compétence.

14 Voir, par exemple, Age Discrimination Act 2004 (Commonwealth), Australian Human Rights Commission Act 1986 (Commonwealth), et Anti-Discrimination Act 1977 (NSW), Equal Opportunity Act 2010 (Vic).

15 Article 345 Fair Work Act 2009 (Commonwealth). 
Si les lois sur la santé et la sécurité au travail dans chacun des six Etats et des deux territoires et au niveau du Commonwealth sont légèrement différentes, elles adoptent toutes le modèle de réglementation "Robens " $^{16}$ et s'appuient fondamentalement sur l'obligation de diligence de l'employeur et des autres parties sur le lieu de travail. Le devoir imposé en vertu de ces lois est que le principal responsable (ce qui a un sens différent selon la juridiction) a le devoir d'assurer la santé et la sécurité du travailleur sur le lieu de travail. Pour ce faire, le responsable en question (y compris l'employeur ou l'entreprise qui exploite un lieu de travail) doit éliminer ou minimiser les risques pour la santé et la sécurité, dans la mesure du possible ${ }^{17}$.

Les lois actuelles sur la santé et la sécurité au travail dans chaque province et territoire ne mentionnent pas spécifiquement le harcèlement professionnel. Cependant, il ressort clairement de l'exposé des motifs de la loi du Commonwealth sur la santé et la sécurité au travail de 2011 que la définition de la santé est destinée à être utilisée " dans son sens le plus large et couvre à la fois la santé physique et psychologique. Cela signifie que la législation couvre les risques psychosociaux pour la santé tels que le stress, la fatigue et le harcèlement ${ }^{18}$ ». C'est donc l'un des risques professionnels que les personnes responsables sont tenues de résoudre et de contrôler «dans la mesure du possible ». Contrevenir à l'un de ces devoirs constitue une infraction pénale. Dans tous les Etats, seuls les inspecteurs des organismes de réglementation de la santé et de la sécurité au travail peuvent enquêter et poursuivre les infractions présumées à la législation pertinente en matière de santé et de sécurité au travail ${ }^{19}$.

En raison de préoccupations au sujet de la capacité des lois sur la santé et la sécurité au travail à traiter les infractions les plus graves ${ }^{20}$, certains Etats ont modifié les lois pénales pour créer une infraction spécifique pouvant aller jusqu'à un comportement d'intimidation très grave ${ }^{21}$.

Les limites du cadre législatif de la santé et de la sécurité au travail ont également été décrites dans le rapport parlementaire Workplace Bullying - We just want it to stop, ce qui a donné naissance à une nouvelle règlementation pour traiter les problèmes de harcèlement dans le travail.

\section{II - LE RAPPORT WORKPLACE BULLYING - WE JUST WANT IT TO STOP (LE HARCĖLEMENT PROFESSIONNEL : NOUS VOULONS JUSTE QUE ÇA S'ARRÊTE)}

En mai 2012, le gouvernement australien a annoncé que le Comité permanent de l'éducation et de l'emploi de la Chambre des représentants mènerait une enquête sur le harcèlement au travail. Le mandat de l'enquête était le suivant : Déterminer s'il existe des lacunes réglementaires, administratives ou inter-juridictionnelles et internationales sur le

16 Nommé d'après le rapport de 1972 du Comité d'enquête britannique présidé par Lord Robens.

17 Article 17 Work Health and Safety Act 2011 (Commonwealth).

18 Explanatory Memorandum to the Work Health and Safety Act 2011 p. 8.

19 A l'exception de la Nouvelle Galles du Sud, où un dirigeant syndical a des pouvoirs similaires.

20 Voir WorkSafe Victoria, Prosecution Results Summaries: Incident Summary: MAP Foundation Pty Ltd 08/02/2010, Victorian WorkCover Authority

http://www1.worksafe.vic.gov.au/vwa/vwa097-002.nsf/content/LSID164635-1>.

21 Par exemple le Crimes Act 1958 (Vic). 
plan juridique et politique qui devraient être corrigées afin d'améliorer la protection contre le harcèlement au travail et d'y réagir rapidement, notamment par des mécanismes de plaintes appropriés ${ }^{22}$.

Lors de la publication du rapport en octobre 2012, le Comité a noté que bien que de nombreux cas d'intimidation psychologique lui aient été rapportés, «il n'y a aucun exemple australien de ces affaires ayant fait l'objet de poursuites devant les tribunaux. Dans les affaires portées devant les tribunaux les atteintes sont physiques et non psychologiques ${ }^{23}$ ». Le titre du rapport est tiré des résultats obtenus par le Comité qui a constaté que $90 \%$ des victimes de harcèlement dans le travail disaient : "Je veux juste que ça s'arrête. Je ne veux pas nécessairement suivre un chemin formel. Je ne veux pas nécessairement de conséquences. Je veux juste que ce comportement prenne fin $^{24} »$.

Le rapport indiquait que le cadre de travail sur la santé et la sécurité était le seul régime légal qui pouvait être appliqué en cas de harcèlement au travail. Comme nous l'avons déjà mentionné, ce cadre ne permet aux autorités d'appliquer la loi que dans le cadre d'une poursuite pénale pour manquement à une obligation de diligence. II s'ensuit que ces cas exigent un haut niveau de preuve et, comme il a été noté précédemment, il y a eu peu de poursuites pour des motifs de harcèlement au travail, particulièrement lorsque le préjudice subi n'a été que psychologique et non physique. De plus, il n'existait aucun mécanisme permettant à un travailleur individuel de demander réparation des conditions subies au travail, au lieu de dépendre du bon vouloir de l'organisme de réglementation qui décidait de l'opportunité des poursuites dans le cadre de ses propres politiques.

La recommandation la plus importante faite dans le rapport, au moins pour les besoins du présent document, était la recommandation 23 : Le Comité recommande que le gouvernement du Commonwealth mette en place des mécanismes permettant aux personnes victimes de harcèlement au travail de bénéficier d'un droit de recours individuel afin d'obtenir réparation dans le cadre d'un processus décisionnel25.

En réponse à la recommandation 23, le gouvernement a présenté le projet de loi sur l'équité dans le travail au Parlement, qui a été adopté et est entré en vigueur le 1 erjanvier 2014. Cette loi a donné lieu à la création d'un nouvel article 6-4B intitulé «Travailleurs victimes de harcèlement au travail » dans le cadre du Fair Work Act de 2009 (Commonwealth) (loi FW).

\section{III - LES DISPOSITIONS SUR LE HARCÈLEMENT AU TRAVAIL ISSUES DU FAIR WORK ACT DE 2009}

La nouvelle compétence a été confiée à la Fair Work Commission (Commission du travail équitable). La Commission est l'héritière d'une institution créée en 1901 sous le nom de Cour de conciliation et d'arbitrage du Commonwealth. Cette juridiction traitait principalement des conflits collectifs entre syndicats et employeurs. La Commission

22 Workplace Bullying: We just want it to stop House of Representatives Standing Committee on Education and Employment, Octobre 2012, p xiii.

23 Workplace Bullying: We just want it to stop House of Representatives Standing Committee on Education and Employment, Octobre 2012, p 5.

24 Workplace Bullying: We just want it to stop House of Representatives Standing Committee on Education and Employment, Octobre 2012 p 95.

25 Workplace Bullying: We just want it to stop House of Representatives Standing Committee on Education and Employment, Octobre 2012 p xiii. 
actuelle est un tribunal indépendant qui traite d'un large éventail de questions relatives aux relations de travail, notamment la mise en place d'un filet de sécurité en matière de salaire minimum et de conditions d'emploi, le contrôle des négociations collectives, la résolution des litiges professionnels collectifs et individuels, la rupture du contrat de travail et d'autres différends fondés sur les droits des salariés.

La Commission est un organe juridictionnel établi en vertu du Fair Work Act et composé de magistrats nommés par le gouvernement et assistés par un personnel administratif. La Commission est chargée des fonctions de règlement extrajudiciaire des différends, comme la médiation et la conciliation, ainsi que des fonctions d'arbitrage qui l'obligent à tirer des conclusions de fait et de droit et à trancher des questions relevant de sa compétence. Les décisions prises par les membres de la Commission engagent les parties en présence, sous réserve des recours en appel devant une assemblée plénière de la Commission et, dans certaines circonstances, d'un contrôle judiciaire par la Cour fédérale d'Australie.

La Commission dispose de pouvoirs très étendus. Elle est guidée, mais non liée, par les règles de preuve et de procédure ${ }^{26}$. Cependant, dans les affaires où elle peut être appelée à exercer son pouvoir décisionnel, la Commission doit appliquer les principes d'équité procédurale et de justice naturelle ${ }^{27}$. Cela signifie que la Commission ne mènera pas d'enquêtes privées et ne s'appuiera que sur des éléments de preuve communiqués à toutes les parties concernées ${ }^{28}$.

En ce qui concerne les pouvoirs spécifiques de la Commission en matière de harcèlement au travail, ils découlent des dispositions suivantes :

L'article 789FC prévoit :

Demande auprès de la FWC d'une ordonnance d'arrêt du harcèlement

(1) Un travailleur qui croit raisonnablement qu'il a été victime d'intimidation au travail peut demander à la FWC une ordonnance en vertu de l'article 789FF.

(2) Aux fins du présent article, le terme de travailleur a le même sens que dans le Work Health and Safety Act de 2011.

L'article 789FD prévoit en outre qu'un travailleur est victime d'un harcèlement professionnel si :

(a) pendant qu'il travaille dans une entreprise visée par la Constitution : (i) un individu ou (ii) un groupe de personnes se livrent de façon répétée à un comportement déraisonnable envers le travailleur ou un groupe dont le travailleur fait partie et (b) ce comportement présente un risque pour la santé et la sécurité.

(2) Pour éviter tout doute, le paragraphe (1) ne s'applique pas aux mesures de gestion raisonnables prises de façon raisonnable.

L'article 789FF prévoit également les éléments à prendre en compte pour que la Commission rende une ordonnance visant à mettre fin à un harcèlement :

La FWC peut rendre des ordonnances pour mettre fin au harcèlement

(1) Si (a) un travailleur a présenté une demande en vertu de l'article 789FC, et (b) la FWC est convaincue que (i) le travailleur a été victime d'un harcèlement au travail perpétré par un individu ou un groupe de personnes, et (ii) il y a un risque que le travailleur continue d'être harcelé au travail par cet individu ou ce groupe,

26 Fair Work Act 2009 s 591.

27 Fair Work Act 2009 s 577.

28 Voir le modèle de gestion des cas d'intimidation disponible sur www.fwc.gov.au 
Alors, la FWC peut rendre toute ordonnance qu'elle juge appropriée (autre qu'une ordonnance exigeant le paiement d'un montant pécuniaire) pour empêcher que le travailleur ne soit victime d'un harcèlement au travail de la part de cet individu ou de ce groupe.

\section{A - LE DROIT DU « TRAVAILLEUR » DE FAIRE UNE DEMANDE}

En vertu des dispositions anti-harcèlement de la Commission, un "travailleur » peut demander à la Commission de rendre une ordonnance visant à mettre un terme au harcèlement s'il croit raisonnablement en avoir été victime ${ }^{29}$. Le terme " travailleur » est défini par référence au Work Health and Safety Act de 2011 (Commonwealth) (ou WHS Act) qui dispose qu'un travailleur est une personne qui exécute un travail à quelque titre que ce soit pour le compte d'une personne ayant une activité ou une entreprise, y compris dans les exemples suivants, un salarié, d'un entrepreneur ou un sous-traitant ; un salarié d'un entrepreneur ou d'un sous-traitant ; un salarié d'une entreprise de location de main-d'œuvre qui a été affecté à un travail dans son entreprise; un travailleur extérieur à l'entreprise ; un apprenti ou un stagiaire ; un étudiant en formation en stage professionnel ; un bénévole - sauf le cas d'une personne qui fait du bénévolat avec une " association de bénévoles » sans salariés.

Sont également définis comme des travailleurs, les membres de la police fédérale australienne (dont le commissaire et le commissaire adjoint) et les titulaires de charges publiques du Commonwealth. Mais la définition ne s'applique pas aux membres des forces de défense australiennes. Diverses décisions de la Commission ont exploré le champ d'application du terme "travailleur ». Ceci a conduit à prendre en compte un soignant s'occupant d'une personne handicapée et qui reçoit le paiement d'une prestation de soignant en vertu de la loi sur la sécurité sociale de 1991 (Commonwealth) ${ }^{30}$, les présidents d'organisations statutaires ${ }^{31}$ et les bénévoles ${ }^{32}$.

\section{B - LA CONDITION D' «ÊTRE AU TRAVAIL »}

Pour que la demande d'un travailleur soit recevable, il doit se trouver " au travail » lorsque le comportement déraisonnable se produit. Le concept selon lequel quelqu'un se trouve « au travail » devient de plus en plus flou, en particulier lorsque les moyens de communication électroniques, y compris le courrier électronique et les réseaux sociaux peuvent être des méthodes utilisées pour se livrer à un comportement déraisonnable. L'expression " au travail » n'est pas définie dans la législation. La même expression est utilisée en relation avec l'obligation de diligence de l'article 19 de la WHS Act et est développée dans son exposé des motifs, selon lequel «le devoir de diligence est lié aux activités professionnelles et ne se limite pas à un lieu de travail physique $»^{33}$.

29 Fair Work Act 2009 s 789FC.

30 Arnold Balthazaarv Department of Human Services (Commonwealth) [2014] FWC 2076, Watson VP, 2 avril 2014.

31 Trevor Yawiriki Adamson [2017] FWC 1976 (Hampton C, 19 mai 2017).

32 Ibid and Gaylene May McDonald [2016] FWC 300 (Hampton C, 15 janvier 2016).

33 Explanatory Memorandum, Work Health and Safety Bill 2011 (Commonwealth) [22]. 
Cette approche générale a été adoptée par la Commission et d'autres juridictions ${ }^{34}$. II est toutefois nécessaire qu'il y ait un lien temporel entre le comportement incriminé et la présence du salarié sur le lieu de travail et cela a été spécifiquement considéré dans le contexte du harcèlement au moyen des réseaux sociaux ${ }^{35}$.

\section{C - Les ENTREPRISES VISÉES PAR LA CONSTITUTION}

Une autre exigence est que le comportement de harcèlement ait lieu pendant que le travailleur est au travail dans une "entreprise visée par la Constitution ». Ceci est motivé par la portée de la Constitution australienne et des pouvoirs conférés au gouvernement du Commonwealth pour l'adoption de certaines lois. Les entreprises concernées comprennent celles qui relèvent des pouvoirs du Commonwealth lui-même, les autorités du Commonwealth, les personnes morales opérant sur un territoire ou dont les affaires ou l'activité sont menées principalement sur un territoire ou sur un lieu du Commonwealth. En ce qui concerne le secteur privé (non gouvernemental), la notion d'« entreprise visée par la Constitution " s'étend à une "société constitutionnelle ", ce qui recouvre les " sociétés étrangères et les sociétés commerciales ou financières constituées dans les limites du Commonwealth ${ }^{36}$ ». Dans la pratique, cela signifie que les dispositions antiharcèlement s'appliquent au secteur privé en Australie et, en termes généraux, n'excluent que les organismes et agences du gouvernement des Etats et certaines administrations locales. Cependant, certaines organisations communautaires non gouvernementales n'ont pas suffisamment d'activités commerciales pour être considérées comme des sociétés commerciales (constitutionnelles) ${ }^{37}$.

\section{D - L'EXISTENCE D'UN À UN COMPORTEMENT RÉPÉTÉ ET DÉRAISONNABLE À L'ENCONTRE DU TRAVAILLEUR}

Le comportement de harcèlement doit être le fait d'un « individu ou d'un groupe de personnes ». Individu signifie une personne physique ${ }^{38}$. Un employeur ou une entreprise ne peut pas, de son propre chef, adopter un comportement d'intimidation au sens du FW Act. De plus, étant donné que le pouvoir de rendre une ordonnance en vertu de l'article 789FF est subordonné à la conclusion qu'il existe un risque que le demandeur continue d'être harcelé au travail par « la » personne ou le groupe de personnes, il sera en général nécessaire que la Commission identifie la personne ou les personnes responsables dans le cadre du processus d'établissement des faits. Toutefois, dans l'affaire Bowker, la Commission a trouvé difficile, compte tenu des éléments dont elle disposait, d'identifier avec précision les personnes qui avaient intimidé les demandeurs. En fin de compte, le groupe de travailleurs a été identifié comme une catégorie de travailleurs déterminée ${ }^{39}$.

34 Clarke $v$ WL Meinhardt and Partners Pty Ltd (non publié, NSW Industrial Court, Fisher CJ, 30 juin 1992), suivant Workcover Authority of NSW (Inspector Farrell) v Ross Colin Morrison [2001] NSWIRC 325 at [50].

35 Bowker and Ors v DP World Melbourne Limited T/A DP Work and Ors [2014] FWCFB (Ross J, Hatch VP, Gostencnik DP, Hampton C, Johns C, 19 décembre 2014, at [55].

36 Commonwealth of Australia Constitution Act 1901 (UK), alinéa 51(xx).

37 Re Ms Marie Pasalskyj [2015] FWC 7309 (Hampton C, 13 novembre 2015), Re Ms Mclnnes [2014] FWC 1395 (Hampton C, 24 mars 2014).

38 Acts Interpretation Act 1901 (Commonwealth) s 2B.

39 [2015] FWC 7312 at [14] (Gostencnik DP). 
La référence aux personnes sur le lieu de travail s'entend du harcèlement non seulement de la part d'autres salariés mais également de personnes qui peuvent être amenées à intervenir sur le lieu de travail telles que les clients ou les visiteurs. Par exemple, une situation, qui est loin d'être rare, c'est la demande faite par un entrepreneur qui fournit des services à des personnes morales dans les complexes résidentiels, comme cela a été le cas dans l'affaire Manderson ${ }^{40}$. Alors que la décision dans cette affaire examinait la question liminaire de savoir si M. Manderson pouvait poursuivre sa demande par l'intermédiaire de la Commission, ce qui est également important à ce sujet c'est que les individus impliqués dans le comportement allégué étaient les propriétaires et occupants de certaines propriétés et non des travailleurs traditionnels sur un lieu de travail.

Afin de répondre à la définition légale de harcèlement, le comportement subi doit être répété, déraisonnable et créer un risque pour la santé et la sécurité. La Commission a conclu que le FW Act devrait être appliqué de la manière suivante :

- Le concept de «comportements répétés » de la part des individus implique l'existence de comportements persistants et déraisonnables, mais pourrait se référer à une série de comportements au fil du temps.

- Un « comportement déraisonnable » doit être considéré comme un comportement qu'une personne raisonnable, compte tenu des circonstances et en y réfléchissant objectivement, peut considérer comme déraisonnable.

- Il doit y avoir un lien de causalité entre le comportement et le risque pour la santé et la sécurité. Le comportement n'a pas besoin d'être l'unique cause, dès lors qu'il s'agit d'une cause substantielle d'un point de vue pratique et selon le bon sens. Le sens ordinaire du terme «risque » est l'exposition au risque de blessure ou de préjudice et doit également être réel et pas simplement éventuel ${ }^{41}$.

Quelques exemples de comportements d'intimidation : remarques grossières et insultes inappropriées, insultes racistes, augmentation de la charge de travail, refus de répondre à une demande d'assistance, rejet d'une demande d'équipement de sécurité, approche inadéquate de la résolution des problèmes de performance et de santé et la conformité à la sécurité ${ }^{2}$, une conduite visant à rabaisser une personne, le fait de jurer, de crier et d'utiliser un langage inapproprié, l'intimidation physique et les menaces de violence ${ }^{43}$.

\section{E - Des mesures de Gestion RAISONNABLES}

La définition du harcèlement indique qu'elle ne s'étend pas aux « mesures de gestion raisonnables prises de manière raisonnable ». Cela a été inclus pour tenir compte du «besoin pour les gestionnaires de pouvoir gérer leur personnel ${ }^{44}$. II s'agit d'un terme

40 Re Manderson [2015] FWC 8231 (Hampton C, 7 décembre 2015).

41 Application by SB [2014] FWC 2104 (Hampton C, 12 May 2014) et Application by Singh [2015] FWC 5850 (Hampton C, 28 August 2015). Voir également la description générale de la compétence dans Amie Mac v Bank of Queensland Limited and Others [2015] FWC 774 (Hatcher VP, 13 février 2015).

42 Bowker and Others v DP World Melbourne Limited T/A DP World and Others [2015] FWC 7312 (Gostencnik DP, 16 novembre 2015).

43 CF and NW [2015] FWC 5272 (Hampton C, 5 août 2015).

44 Fair Work Amendment Bill Explanatory Memorandum p 29. 
commun considéré comme faisant partie des lois sur l'indemnisation des travailleurs, signalées précédemment, qui exempte de l'obligation d'indemnisation dans les cas où un salarié a subi des blessures psychologiques à la suite de "mesures administratives raisonnables (ou de gestion) menées de façon raisonnable ». Les mesures de gestion qui ne sont pas raisonnables ou qui ne sont pas appliquées de façon raisonnable pourraient tout de même être considérées comme du harcèlement. Comme c'est le cas pour le harcèlement, d'une manière plus générale, ce concept est considéré par la Commission sur une base objective dans le contexte des parties concernées ${ }^{45}$.

Dans l'affaire Burbeck v Alice Springs Town Council la Commission a examiné de près chaque décision managériale que la requérante a déclaré ne pas être une mesure de gestion raisonnable menée de manière raisonnable ${ }^{46}$. Ce faisant, la Commission a estimé que certaines mesures raisonnables de la part de la direction n'étaient pas raisonnables, y compris le fait que l'employeur n'ait pas tenu compte des griefs déposés par la requérante en réponse à certaines des mesures prises contre elle. Pour déterminer s'il y avait lieu d'émettre des ordonnances, la Commission a estimé qu'il était probable que le comportement déraisonnable persisterait et qu'il y avait un risque que cela puisse créer un danger pour la santé et la sécurité. Le caractère continu du comportement a été en partie attribué à la requérante, considérant que «la présence de ce comportement contributif et de la conduite tempère inévitablement la considération de ce qui peut être fait par rapport à ce comportement ». Les ordonnances rendues par le commissaire prévoyaient la mise en place d'une formation en matière de lutte contre le harcèlement et de communication positive qui devait être suivie par toutes les personnes concernées par la demande, y compris la requérante.

\section{F - L'EXISTENCE D'UN RISOUE POUR LA SANTÉ ET LA SÉCURITÉ D'UN RISOUE CONTINU DE HARCÈLEMENT AU TRAVAIL}

Pour permettre à la Commission d'ordonner l'arrêt du harcèlement, il faut s'assurer que le travailleur a été intimidé au travail par un individu ou un groupe de personnes et qu'il y a un risque que le travailleur continue d'être victime d'un harcèlement au travail de la part des individus concernés ${ }^{47}$. Par conséquent, la Commission ne peut émettre une ordonnance que si le travailleur continue d'être victime d'intimidation au travail et ne peut pas émettre une ordonnance si le risque n'existe plus, comme cela peut être le cas si le travailleur n'est plus au travail parce qu'il a démissionné ou a été licencié. Cette situation se présente parfois et constitue une limite au fonctionnement pratique de la juridiction. De plus, si l'employeur prend des mesures appropriées pour éliminer le risque que le harcèlement ne se poursuive ${ }^{48}$, ou si les personnes concernées ont quitté le lieu de travail, la Commission n'a aucune raison d'ordonner la cessation du harcèlement.

45 Re Ms SB [2014] FWC 2104 (Hampton C, 12 mai 2014) para. 48.

46 Burbeck v Alice Springs Town Council, Georgina Davison, Skye Price, Clare Fisher [2017] FWC 4988 (Wilson C, 6 octobre 2017).

47 Fair Work Act 2009 (Commonwealth) s 789FF. Voir également Atkinson v Killarney Properties Pty Ltd T/A Perm-A-Pleat Schoolwear and Michael Palm [2015] FWCFB 6503 (Acton SDP, Gooley DP, Roe C, 14 octobre 2015) para. 35.

48 Re Ms LP [2015] FWC 6602 (Hampton C, 4 November 2015). 


\section{G - Les ORdonNANCES DE LA COMMISSION}

Si la Commission est convaincue qu'un travailleur a été victime d'un harcèlement et qu'il existe un risque futur, elle peut alors rendre toute ordonnance préventive jugée appropriée. La Commission dispose de larges pouvoirs pour prendre les mesures préventives qu'elle juge appropriées. Ces pouvoirs doivent être éclairés par la conduite déraisonnable antérieure, telle qu'elle a été établie, sans toutefois s'y limiter. Cependant, toute ordonnance doit viser à empêcher le travailleur d'être harcelé à l'avenir par l'individu ou le groupe concerné, être fondée sur des constatations appropriées et tenir compte des considérations établies par l'article 789FF (2) du FW Act ${ }^{49}$. La loi interdit toutefois à la Commission de rendre une ordonnance d'indemnisation ou de condamner l'employeur à verser une somme d'argent. La Commission peut également rendre des ordonnances provisoires, dans des circonstances exceptionnelles, notamment pour assurer la sécurité des parties ou pour permettre à la demande d'être entendue sur le fond ${ }^{50}$.

La plupart des questions soumises à la Commission sont résolues suite à l'intervention de la Commission et sans qu'aucune ordonnance ne soit rendue. Depuis l'attribution de cette compétence, le nombre de demandes présentées est demeuré relativement stable, soit 722 en 2016-2017, 734 en 2015-2016 et 694 en 2014-2015 51 . Le nombre de demandes entraînant une décision, c'est-à-dire celles qui sont effectivement déterminées par la Commission, représente un pourcentage relativement faible de la totalité de ces demandes. Par exemple, en 2016-2017, seulement 60 demandes, soit $9 \%$ de toutes les questions réglées, ont été finalisées par une décision. Sur ce total, seule une petite proportion des affaires concernait des ordonnances passées par la Commission. Ceci est le résultat des diverses conditions juridictionnelles préalables, de l'obligation pour le travailleur demandeur d'être encore présent sur le lieu de travail dans le cadre de toute ordonnance d'interdiction de harcèlement et des taux de résolution élevés ${ }^{52}$.

Pour conclure, la jurisprudence australienne relative au harcèlement au travail s'est considérablement développée ces derniers temps avec l'adoption du Fair WorkAmendment Act de 2013. Auparavant, le cadre législatif était relativement stable, mais peu de décisions de justice aidaient efficacement à son interprétation. Ce qui a été démontré, c'est que la jurisprudence australienne relative au harcèlement au travail s'est développée de telle sorte que, jusqu'à récemment, la réparation ne pouvait être recherchée ou poursuivie que lorsqu'un cas de harcèlement très grave s'étai produit, souvent avec des conséquences très sérieuses. La nouvelle compétence de la Commission vise à essayer d'arrêter ce harcèlement avant qu'il n'entraîne de telles conséquences. Bien que cette compétence ne soit pas nécessairement appropriée ou disponible pour tous les cas de harcèlement, il s'agit d'une compétence supplémentaire qui sensibilise le public à la question et permet de répondre rapidement aux préoccupations immédiates de nombreux travailleurs qui croient être victimes de harcèlement au travail ${ }^{53}$.

49 Churches et al [2016] FWCFB 2367 (O'Callaghan SDP, Clancy DP, Hampton C, 14 avril 2016) para 32.

50 Re Bayly [2017] FWC 1886, (Hampton C, 5 avril 2017).

51 Fair Work Commission, rapport annuel 2016-17, p 96.

52 Par exemple, en 2016-17 188 demandes ont été directement résolues au cours de la procédure, Fair Work Commission, rapport annuel 2016-17, p 96.

53 Voir Department of Employment - Post Implementation Review - février 2017, http://ris.pmc.gov.au/2017/02/09/changes-fair-work-act-2009 